\title{
COMUNIDADE ZOOPLANCTÔNICA EM UM PEQUENO CORPO D'ÁGUA ASSOCIADO A UM FRAGMENTO FLORESTAL E PASTAGEM NO MUNICÍPIO DE ALFENAS - MG
}

Paula Nunes Coelho ${ }^{1}$

\section{Eugênio Bastos Bernardes de Oliveira ${ }^{2}$}

\author{
Maria José dos Santos-Wisniewski ${ }^{3}$
}

\begin{abstract}
RESUMO
O objetivo deste estudo foi realizadar um levantamento da comunidade zooplanctônica em um pequeno corpo d'água raso com macrófitas aquáticas, com um fragmento florestal em uma de suas margens e pastagens na outra, localizado no município de Alfenas-MG. As amostras do zooplâncton foram obtidas por meio de arrastos horizontais a partir da margem do corpo d'água, nos meses de junho, julho, outubro e novembro de 2012. As variáveis físicas e químicas (temperatura, condutividade elétrica, $\mathrm{pH}$, oxigênio dissolvido e sólidos totais dissolvidos) da água foram medidas in situ e foram coletadas amostras de água para determinação de clorofila a e material em suspensão. Os resultados das variáveis físicas e químicas evidenciaram uma variação sazonal com aumento nas temperaturas da água, condutividade elétrica e nos sólidos totais dissolvidos. A comunidade zooplanctônica foi representada por 50 espécies distribuídas entre Cladocera, Copepoda e Rotifera, com grande riqueza de espécies de Cladocera da família Chydoridae (15 espécies) enquanto entre o Rotifera destacaram as famílias Brachionidae (7) e Lecanidae (7). As espécies mais abundantes no corpo d'água foram Chydorus pubescens, Acroperus tupinamba e llyocryptus spinifer para os cladóceros, Conochilus unicornis, Plationus patulus e Lecane bulla para os rotiferos. Para o Copepoda houve o predomínio das formas jovens (náuplios e copepoditos) de Cyclopoida. Embora o corpo d'água apresente um pequeno tamanho, pequena profundidade e atividades antrópicas no entorno, conclui-se que esse ecossistema abriga uma elevada diversidade de espécies mesmo com ocorrência de espécies típicas de ambientes com maior grau de trofia.
\end{abstract}

PALAVRAS-CHAVE: Açude. Zooplâncton. Região litorânea.

\footnotetext{
${ }^{1}$ Bióloga, Programa de Pós-Graduação em Ecologia e Tecnologia Ambiental, Universidade Federal de Alfenas-MG. paulinhancoelho@gmail.com

${ }_{2}^{2}$ Biólogo, Universidade Federal de Alfenas-MG. maracatueugenio@yahoo.com.br

3 Professora, Instituto Ciências da Natureza, Universidade Federal de Alfenas-MG.cwz@uol.com.br
} 


\title{
ZOOPLANKTON COMMUNITY BODY IN A SMALL WATER ASSOCIATED WITH A SHRED FOREST AND GRASSLAND IN ALFENAS COUNTY - MG
}

\begin{abstract}
The objective of this study was to survey the zooplankton community in a small body of shallow water with aquatic weeds, with a forest fragment in one of its banks and pastures in the other, located in the city of Alfenas-MG. Zooplankton samples were obtained by horizontal hauls from the edge of the water body in the months of June, July, October and November 2012. The physical and chemical variables (temperature, conductivity, $\mathrm{pH}$, dissolved oxygen and total dissolved solids) of water in situ measurements and water samples were collected for determination of chlorophyll a and suspended matter. The results of the physical and chemical variables showed a seasonal variation with increase in water temperatures, electrical conductivity and total dissolved solids. The zooplankton community was represented by 50 species distributed among Cladocera, Copepoda and Rotifera, with rich Cladocera species of Chydoridae family (15 species) while among the Rotifera highlighted the Brachionidae families (7) and Lecanidae (7). The most abundant species in the water body were Chydorus pubescens, Acroperus tupinamba and llyocryptus spinifer for cladocerans, unicornis Conochilus, Plationus patulus and Lecane bulla for rotifers. For Copepoda was the predominance of young forms (nauplii and copepodites) Cyclopoida. Although the body of water with a small, shallow and human activities on the environment, it is concluded that this ecosystem is home to a high diversity of species, even with the occurrence of typical species environments with higher trophic level.
\end{abstract}

KEYWORDS: Weir. Zooplankton. Coastal region

\section{EL ZOOPLANCTON ORGANISMO COMUNITARIO EN UNA PEQUEÑA AGUA ASOCIADAS CON UN FRAGMENTO DE BOSQUE Y PASTIZALES EN ALFENAS CONDADO - MG}

\begin{abstract}
RESUMEN
El objetivo de este estudio fue realizadar una encuesta de la comunidad de zooplancton en un pequeño cuerpo de agua poco profunda con malezas acuáticas, con un fragmento de bosque en una de sus orillas y pastos en el otro, que se encuentra en la ciudad de Alfenas-MG. Las muestras de zooplancton se obtuvieron mediante lances horizontal desde el borde de la masa de agua en los meses de junio, julio, octubre y noviembre de 2012. Las variables físicas y químicas (temperatura, conductividad, $\mathrm{pH}$, oxígeno disuelto y sólidos disueltos totales) de agua en las mediciones in situ y tomaron muestras de agua para la determinación de clorofila y materia en suspensión. Los resultados de las variables físicas y químicas mostraron una variación estacional con el aumento de las temperaturas del agua, conductividad eléctrica y sólidos disueltos totales. La comunidad de zooplancton estuvo representada por 50 especies distribuidas entre Cladocera, copépodos y rotíferos, con ricas especies Cladocera de familia Chydoridae (15 especies), mientras que entre los rotiferos destacó las familias Brachionidae (7) y Lecanidae (7). La especie más abundante en el cuerpo de agua eran pubescens Chydorus, Acroperus tupinamba y llyocryptus spinifer de cladóceros, unicornis Conochilus, Plationus patulus y Lecane bulla de rotíferos. Para Copepoda fue el predominio de las formas jóvenes (nauplios y copepoditos) Cyclopoida. Aunque el cuerpo de agua con un pequeño, de poca profundidad y las actividades humanas sobre el medio ambiente, se concluye que este ecosistema es el hogar de una gran diversidad de especies, incluso con la aparición de entornos de especies típicas de mayor nivel trófico.
\end{abstract}

PALABRAS-CLAVE: Weir. El zooplancton. Región costera. 


\section{INTRODUÇÃO}

Os pequenos corpos d'água são importantes para a conservação da biodiversidade, pois são ambientes capazes de abrigar uma alta diversidade, com espécies raras e únicas em relação aos corpos d'água de maior tamanho (WILLIAMS et al., 2003; DAVIES et al., 2008). Embora estes sistemas aquáticos de água doce estejam presentes em abundância na ecologia de paisagem, grande parte dos estudos são centrados nos sistemas aquáticos de grande porte como os reservatórios (CASTILHO-NOLL et al., 2012; NEVES et al., 2013; BEAVER et al., 2014), rios (MASSICOTTE et al., 2014; DICKERSON et al., 2010) e grandes lagos (SYMONS et al., 2014). Dessa forma, os pequenos corpos d'água atuam como "bolsões" de biodiversidade diferenciando dos outros sistemas maiores (LEON et al., 2010).

Estes ambientes possuem uma natureza insular e são considerados como "ilhas aquáticas" em uma paisagem terrestre (DE MEESTER, 2005). O ambiente tem contato direto e mais próximo com os ecossistemas terrestres adjacentes e carecem de um verdadeiro sistema pelágico (PALIK et al., 2001). A pequena profundidade permite a penetração de luz até o fundo do corpo d'água o que resulta no desenvolvimento de macrófitas (DECLERCK et al., 2006) e maior acoplamento bentônico-pelágico (SØNDERGAARD et al., 2005). Estes pequenos elementos da paisagem cumprem uma importante função ecológica, pois favorecem a permeabilidade para biota na paisagem (MERRIAM, 1991; DE MEESTER et al., 2005).

No entanto, apesar da importância ecológica dos pequenos corpos d'água, a biodiversidade desses ecossistemas esta ameaçada pelas pressões externas tais como poluição, eutrofização, introdução de espécies exóticas, alteração e/ou destruição de habitats resultando no declínio da diversidade de espécies (TUNDISI, 2003; ROCHA et al., 2005).

Das comunidades presentes nesses ecossistemas, o zooplâncton é muito diversificado, com função determinante na transferência de energia dos produtores primários para os consumidores de níveis tróficos superiores e como bioindicadores 
da qualidade das águas (MAJER, 1987; ZANNATUL; MUKTADIR, 2009). A comunidade zooplanctônica é composta por: Rotífera, duas subclasses de Crustacea (Cladocera e Copepoda), protozoários e por outros representantes menos frequentes como o Ostracoda, Turbellaria, ácaros e larvas de insetos (WETZEL, 1993).

A zona litorânea abriga uma riqueza elevada em relação à zona pelágica principalmente quando são ocupadas por vegetação aquática, favorecendo assim a diversidade de espécie nessa região (MARGALEF, 1983; HAVENS, 1991; LIMA et al., 2003; VAN ONSEM et al., 2010). Essa alta diversidade local ocorre devido à elevada heterogeneidade ambiental gerada pelo desenvolvimento de macrófitas que fornecem ao zooplâncton abrigo e refúgio, proporcionando diversos micro-habitats (TOWNSEND et al., 2010; VAN DONK; VAN DE BUND, 2002). A maioria das espécies que habita a vegetação são organismos fitófilos, como os cladóceros das famílias Chydoridae e Macrothricidae. (ELMOOR-LOUREIRO, 2007).

Considerando a contribuição dos corpos d'água menores para a diversidade de espécies, o presente estudo visou realizar um levantamento da comunidade zooplanctônica em um corpo d' água pequeno, raso, com um fragmento florestal em uma de suas margens e pastagens na outra, para contribuir com novas informações sobre a biodiversidade do zooplâncton no estado de Minas Gerais.

\section{1. Área de estudo}

O estudo foi desenvolvido em um pequeno corpo d'água (açude), formado pelo represamento de um córrego proveniente de uma nascente dentro de uma propriedade rural denominada Fazenda Matão, no município de Alfenas, no sul do Estado de Minas Gerais, Brasil (2130'22”S; 4552'57'W) (Figura 1).

O clima da região é do tipo Cwb (clima subtropical moderado úmido), com invernos secos e verões chuvosos, de acordo com o sistema de Köppen. A temperatura anual média é de cerca de $23^{\circ} \mathrm{C}$ e a precipitação média anual em torno de $1500 \mathrm{~mm}$. O açude esta localizado em uma altitude de 780 metros, com predomínio de relevo montanhoso (COSTA et al., 1998; MELLO et al., 2003). 
O corpo d'água possui uma de suas margens associada, em sua maior parte, por um fragmento florestal de Mata Atlântica Estacional Semidecidual e na outra por pastagem com presença de bovinos. O volume de água desse ambiente é influenciado pela precipitação local, dessa forma, o corpo d'água esta sujeito a períodos de cheia, quando há elevados valores pluviométricos entre os meses de novembro a abril, e com diminuições nos níveis da água, durante a estação seca de maio a outubro. Este ambiente é usado para dessedentação animal, pesca e abastecimento de água em residências e agricultura.

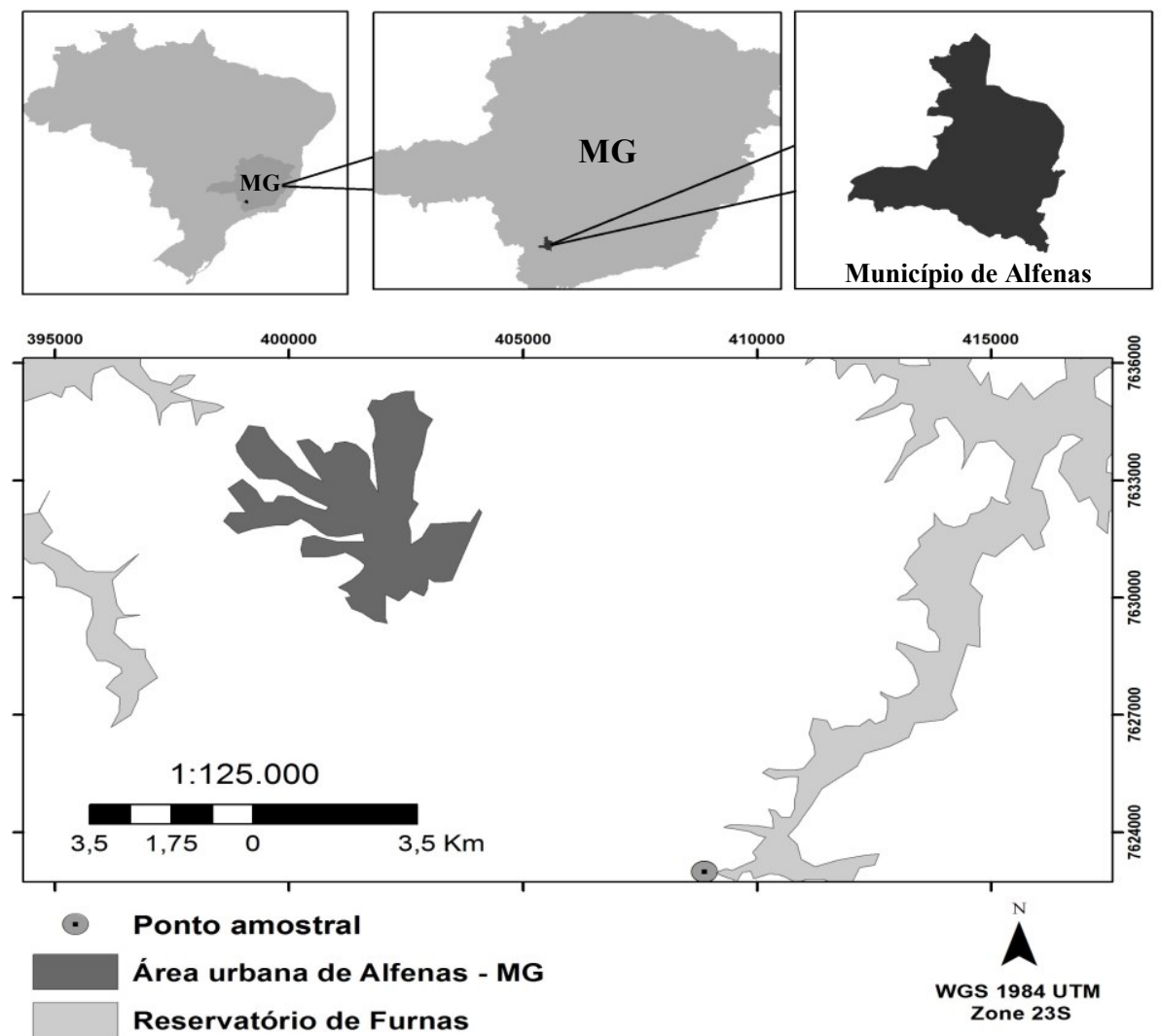

Figura 1- Mapa com a localização do açude da Fazenda Matão no município de Alfenas do estado de Minas Gerais.

\section{MATERIAIS E MÉTODOS}

As amostragens foram realizadas nos meses de junho e julho/2012 (estação seca) e outubro e novembro do mesmo ano (estação chuvosa) a partir de um ponto fixo da margem do corpo d'água.

A determinação da temperatura da água, da concentração de oxigênio dissolvido, da condutividade elétrica, dos sólidos totais dissolvidos e do $\mathrm{pH}$ foi 
realizada na superfície da água com um multisensor Horiba U-22. A concentração do material em suspensão foi determinada pelo método gravimétrico descrito por Teixeira et al., (1965). As determinações da concentração de clorofila a foram realizadas pelo método de extração com acetona 90\% (GOLTERMAN et al., 1978). As coletas de zooplâncton para a análise qualitativa foram realizadas por meio de arrastos horizontais na coluna d'água com uma rede de plâncton com abertura de malha $68 \mu \mathrm{m}$. Para amostras quantitativas, devido à pequena profundidade do corpo d'água (< 2 metros), foram filtrados 100 litros de água, com um balde de 10 litros e passados pela rede de plâncton. O material coletado foi fixado com formol em concentração final de $4 \%$, saturado com açúcar. As contagens e identificação de Cladocera, Copepoda e Rotifera foram realizadas em microscópio estereoscópio (aumento até 50x) e microscópio óptico (aumento até 1000x). A identificação dos organismos foi feita utilizando-se bibliografia especializada (ELMOOR-LOUREIRO, 1997; ELMOOR-LOUREIRO, 2010; DE MELO; HEBERT, 1994; SINEV; ELMOORLOUREIRO, 2010; SMIRNOV, 1974; KOSTE, 1978, KOTOV; DUMONT, 2000; SEGERS, 1995; SEGERS, 2007). O material amostrado foi depositado na coleção de amostras do Laboratório de Limnologia da UNIFAL-MG, Brasil.

\section{RESULTADOS E DISCUSSÕES}

O corpo d'água em estudo é um ecossistema de pequena dimensão (área de $8118,12 \mathrm{~m}^{2}$ ), raso e colonizado por macrófitas aquáticas do gênero Nymphaea sp. A temperatura da água foi mais baixa em julho $\left(20,73^{\circ} \mathrm{C}\right)$ e mais alta em novembro $\left(27,02^{\circ} \mathrm{C}\right)$, com concentrações de oxigênio dissolvido variando de $4,6 \mathrm{mg} \mathrm{L}^{-1} \mathrm{em}$ junho e a $7,8 \mathrm{mg} \mathrm{L}^{-1}$ registrado em outubro de 2012. $\mathrm{O} \mathrm{pH}$ do corpo d'água foi ligeiramente ácido a neutro com valores superiores a 5,7 (Tabela 1). A diminuição das concentrações de oxigênio dissolvido e do $\mathrm{pH}$ associado com aumento da temperatura em novembro pode ser evidenciados pela decomposição da vegetação aquática que consome parte do oxigênio presente na água (HENRY et al., 2005).

Os valores de sólidos totais dissolvidos foram maiores em outubro $\left(0,052 \mathrm{~g} \mathrm{~L}^{-1}\right)$ e novembro $\left(0,066 \mathrm{~g} \mathrm{~L}^{-1}\right)$. A condutividade elétrica da água variou de 50 a $100 \mu \mathrm{S}$ $\mathrm{cm}^{-1}$ e a clorofila a de 6,06 a $19,55 \mu \mathrm{g} \mathrm{L} \mathrm{L}^{-1}$. O aumento na concentração de sólidos 
totais dissolvidos, condutividade elétrica e concentração de clorofila a em outubro e novembro reflete a entrada de material alóctone ocasionado pelas chuvas que consequentemente contribuiu para o crescimento do fitoplâncton. A radiação solar e temperatura são outros fatores limitantes responsáveis por aumentar a produtividade nos ambientes aquáticos (TOWNSEND et al., 2010).

Apesar das concentrações de sólidos totais dissolvidos evidenciarem um aumento na estação chuvosa, os valores da concentração de material em suspensão com predomínio de matéria orgânica, no geral foram baixos (4,7- 6,4 mg $\mathrm{L}^{-1}$ ) comparados ao Reservatório do Monjolinho em São Carlos onde foram registrados valores entre $37-70 \mathrm{mg} \mathrm{L}^{-1}$ do material em suspensão (SANTOS et al., 2011). As baixas concentrações de material em suspensão podem estar relacionadas à presença de bancos de macrófitas aquáticas que retêm parte do material em suspensão (CABRAL et al., 2009).

Tabela 1. Os valores obtidos para as variáveis físicas, químicas e biológicas do corpo d' água da Fazenda Matão no período de estudo, em 2012. OD = oxigênio dissolvido; CE = condutividade elétrica; STD = sólidos totais dissolvidos; $\mathrm{MO}$ = matéria orgânica; $\mathrm{MI}$ = matéria inorgânica.

\begin{tabular}{ccccc}
\hline Variáveis & Junho & Julho & Outubro & Novembro \\
\hline Temperatura da água $\left({ }^{\circ} \mathrm{C}\right)$ & 26,93 & 20,73 & 23,2 & 27,02 \\
OD $\left(\mathrm{mg} \mathrm{L}^{-1}\right)$ & 4,6 & 6,45 & 7,8 & 6,7 \\
Cond. $\left(\mu \mathrm{S} \mathrm{cm}^{-1}\right)$ & 50 & 60 & 80 & 100 \\
STD $\left(\mathrm{g} \mathrm{L}^{-1}\right)$ & 0,033 & 0,036 & 0,052 & 0,066 \\
$\mathrm{pH}$ & 6,34 & 7,72 & 6,36 & 5,7 \\
$\mathrm{MO}\left(\mathrm{mg} \mathrm{L}^{-1}\right)$ & 2,4 & 2,6 & 3,7 & 3,8 \\
$\mathrm{Ml}\left(\mathrm{mg} \mathrm{L}^{-1}\right)$ & 2,3 & 1,9 & 2,7 & 1,6 \\
Clorofila $a\left(\mu \mathrm{g} \mathrm{L}^{-1}\right)$ & 6,06 & 17,52 & 19,55 & 19,55 \\
\hline
\end{tabular}

O zooplâncton esteve representado por um total de 50 espécies, distribuídas entre cladóceros (25) e rotíferos (25). Os copépodos não foram identificados, impossibilitando a quantificação de espécies nesse grupo (Tabela 2, 3 e 4).

Tabela 2. Densidade numérica (org. $\mathrm{m}^{-3}$ ) e frequência de ocorrência de espécies de Cladocera no corpo d'água da Fazenda Matão nos meses de junho, julho, outubro e novembro de 2012.

\begin{tabular}{rccccc}
\hline Cladocera & Jun. & Jul. & Out. & Nov. & $\begin{array}{c}\text { Frequência } \\
(\%)\end{array}$ \\
\hline Acroperus tupinamba & 90 & 3120 & 350 & 220 & 100
\end{tabular}




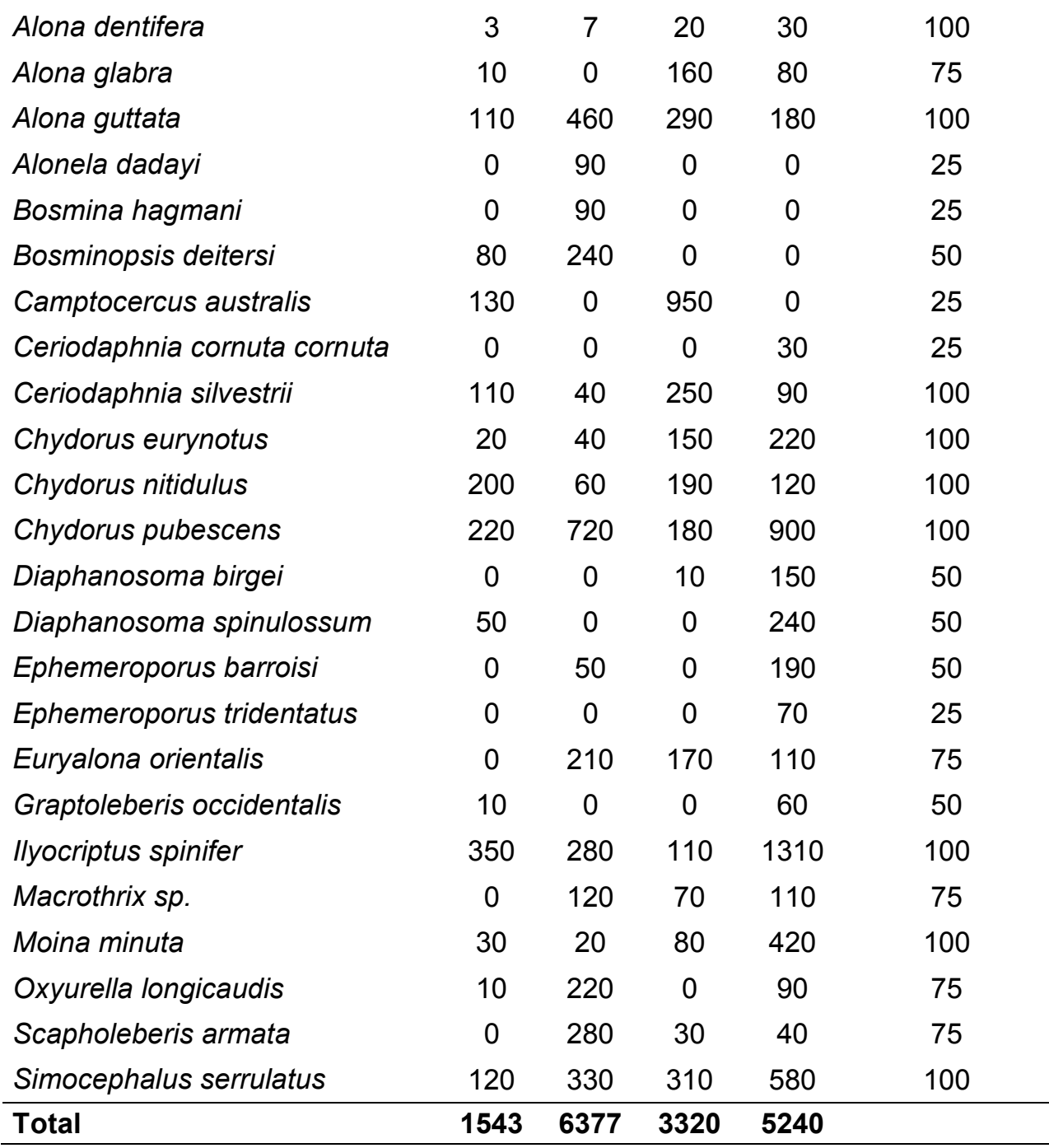

Tabela 3 - Densidade numérica (org. $\mathrm{m}^{-3}$ ) e frequência de ocorrência de Copepoda no açude da Fazenda Matão nos meses de junho, julho, outubro e novembro de 2012.

\begin{tabular}{cccccc}
\hline Copepoda & Jun. & Jul. & Out. & Nov. & Frequência (\%) \\
\hline Copepoda Calanoida & 20 & 20 & 0 & 20 & 75 \\
Adulto & & & 210 & 40 & 100 \\
Copepodito Calanoida & 700 & 1750 & 2100 & 100 \\
Náuplios Calanoida & 1000 & 1580 & 340 & 440 & 75 \\
Copepoda Cyclopoida & 320 & 0 & 140 & 180 & 100 \\
Adulto & & & & & 100 \\
Copepodito Cyclopoida & 870 & 2110 & 2410 & 3880 & \\
Náuplios Cyclopoida & 1870 & 2900 & 2460 & 640 & \\
\hline Total & $\mathbf{4 7 8 0}$ & $\mathbf{8 3 6 0}$ & $\mathbf{5 5 6 0}$ & $\mathbf{5 2 0 0}$ & \\
\hline
\end{tabular}


Tabela 4. Densidade numérica (org. $\mathrm{m}^{-3}$ ) e frequência de ocorrência das espécies de Rotifera no açude da Fazenda Matão nos meses de junho, julho, outubro e novembro do ano de 2012.

\begin{tabular}{lccccc}
\hline Rotifera & Jun. & Jul. & Out. & Nov. & $\begin{array}{c}\text { Frequência } \\
\text { (\%) }\end{array}$ \\
\hline Anuraeopsis. sp & 0 & 0 & 0 & 40 & 25 \\
Brachionus dolabratus & 0 & 0 & 220 & 70 & 50 \\
Conochilus coenobasis & 0 & 0 & 0 & 120 & 25 \\
Conochilus unicornis & 0 & 10 & 7087 & 80 & 75 \\
Euchlanis dilatata & 0 & 80 & 0 & 200 & 50 \\
Euchlanis lyra & 0 & 70 & 0 & 0 & 25 \\
Hexartra intermedia & 0 & 0 & 1260 & 220 & 50 \\
Kellicotia bostoniensis & 160 & 2190 & 0 & 20 & 75 \\
Keratella cochlearis & 260 & 20 & 0 & 20 & 75 \\
Keratella lenzi & 50 & 0 & 0 & 20 & 50 \\
Lecane bulla & 310 & 190 & 310 & 1100 & 100 \\
Lecane closterocerca & 0 & 0 & 0 & 20 & 25 \\
Lecane cornuta & 0 & 80 & 0 & 120 & 50 \\
Lecane curvicornis & 0 & 50 & 30 & 40 & 75 \\
Lecane furcata & 0 & 0 & 0 & 110 & 25 \\
Lecane leontina & 20 & 40 & 0 & 120 & 75 \\
Lecane monostyla & 0 & 0 & 0 & 120 & 25 \\
Lepadella patella & 90 & 100 & 0 & 100 & 75 \\
Platyias quadricornis & 0 & 70 & 0 & 300 & 50 \\
Plationus patulus & 210 & 700 & 0 & 920 & 75 \\
Polyarthra vulgaris & 30 & 0 & 0 & 220 & 50 \\
Testudinella patina & 10 & 0 & 0 & 0 & 25 \\
Trichocerca bicristata & 0 & 10 & 0 & 140 & 50 \\
Trichocerca cylindrica & 400 & 120 & 30 & 0 & 75 \\
Trichotria tetractis & 0 & 10 & 0 & 40 & 50 \\
\hline \multicolumn{1}{c}{ Total } & 1540 & $\mathbf{3 7 4 0}$ & $\mathbf{8 9 3 7}$ & $\mathbf{4 1 4 0}$ & \\
\hline & & & & & \\
\hline
\end{tabular}

O número de táxons encontrados no açude em estudo revelou uma alta riqueza de espécies. Considerando que o esforço de amostragem foi baixo e o corpo d'água possui uma pequena dimensão, o número de táxons registrados reflete a diversidade de espécies da área. Esse resultado pode ser uma consequência da baixa profundidade e do pequeno tamanho do corpo d'água que resulta em um ambiente favorável para a colonização de macrófitas. Portanto a presença dessa vegetação proporciona maior heterogeneidade ambiental o que contribuiu para uma 
grande diversidade de espécies (VAN ONSEM et al., 2010; TOWNSEND et al., 2010).

Alguns autores (NUNES et al., 1996; MAIA-BARBOSA et al., 2008; CASTILHONOLL et al., 2012) também encontraram uma elevada diversidade de espécies do zooplâncton em ambientes colonizados por macrófitas aquáticas. Os resultados do presente estudo corrobora com o estudo desenvolvido por Maia-Barbosa et al., (2014) que também encontraram uma maior diversidade de espécies em um lago pequeno e raso com presença de macrófitas.

Para os cladóceros, a família com maior riqueza de espécie foi Chydoridae com um total de 15 espécies e Daphnidae com 5. De acordo com Elmoor-Loureiro (2007), uma elevada riqueza de espécies dessa família é característica de ambientes dominados por macrófitas, pois estas espécies utilizam para a alimentação apêndices especializados na raspagem de substratos. Santos-Wisniewski et al., (2002) também encontraram alta riqueza de espécies de Chydoridae associada a presença de macrófitas em pequenos corpos d'água de grande altitude na Serra da Mantiqueira-SP. Uma revisão sobre o levantamento de espécies de Cladocera nas águas doces do Estado de Minas Gerais confirma que o predomínio da família Chydoridae representa a maior riqueza de espécies no estado, o equivalente a $50 \%$ da riqueza total dos Cladocera amostrados (SANTOS-WISNIEWSKI et al., 2011).

Entre as espécies de cladóceros encontrados, Chydorus pubescens e Acroperus tupinamba foram os dominantes com maiores densidades (Tabela 2). Chydorus pubescens também teve ampla distribuição geográfica no inventário de distribuição das espécies do gênero Chydorus e Pseudochydorus em diversos corpos d'água do estado de São Paulo (SANTOS-WISNIEWSKI et al., 2008). Para Acroperus tupinamba, Sinev; Elmoor-Loureiro (2010) relatam que esta pode ser uma espécie críptica de Acroperus harpae. Segundo esses autores, a ocorrência de Acroperus harpae no Brasil provavelmente refere-se ao Acroperus tupinamba. Esta espécie foi considerada com alta ocorrência em corpos d'água avaliados no Distrito Federal (SOUZA; ELMOOR-LOUREIRO, 2013) e possui uma ampla distribuição geográfica no país (SOUZA; ELMOOR-LOUREIRO, 2008). 
llyocryptus spinifer foi observado com elevada densidade e frequência de ocorrência no açude. A elevada densidade e frequência dessa espécie no estudo são resultantes da pequena profundidade do corpo d'água uma vez que essa espécie possui hábitos bentônicos e ocorrência em ambientes colonizados por macrófitas aquáticas (SOUZA; ELMOOR-LOUREIRO, 2008; SOUZA et al., 2013). Além disso, durante a amostragem, o fundo do corpo d'água pode ter atingido e influenciado na densidade desta espécie.

Os rotíferos foram representados por 11 famílias sendo a Brachionidae ( 7 espécies) e Lecanidae (7) predominantes no estudo. O predomínio dessas famílias também foi relatado por Bonecker et al., (1994) e Serafim et al., (2003). A riqueza de espécies de Lecanidae está associada à ocorrência de vegetação aquática, pois as espécies dessa família apresentam hábitos litorâneos (SEGERS, 1996). As espécies de Rotifera, Plationus patulus e Lecane bulla contribuíram com alta densidade de indivíduos durante o estudo enquanto Conochilus unicornis teve alta densidade no mês de outubro. Segundo Matsumura-Tundisi et al., (1990), Conochilus unicornis é um forte indicador do processo de eutrofização no reservatório de Barra Bonita.

A abundância relativa de Copepoda foi maior nos meses de junho e julho enquanto em outubro o Rotifera foi dominante (Figura 2). Em novembro, houve uma distribuição mais uniforme dos grupos zooplanctônicos. A dominância de Rotifera no mês de outubro pode ser explicada pela reprodução partenogenética, curtos ciclos de vida e a produção de ovos resistentes que favorece o sucesso do grupo em ambientes tropicais (NEVES et al., 2003; MAIA-BARBOSA et al., 2014).

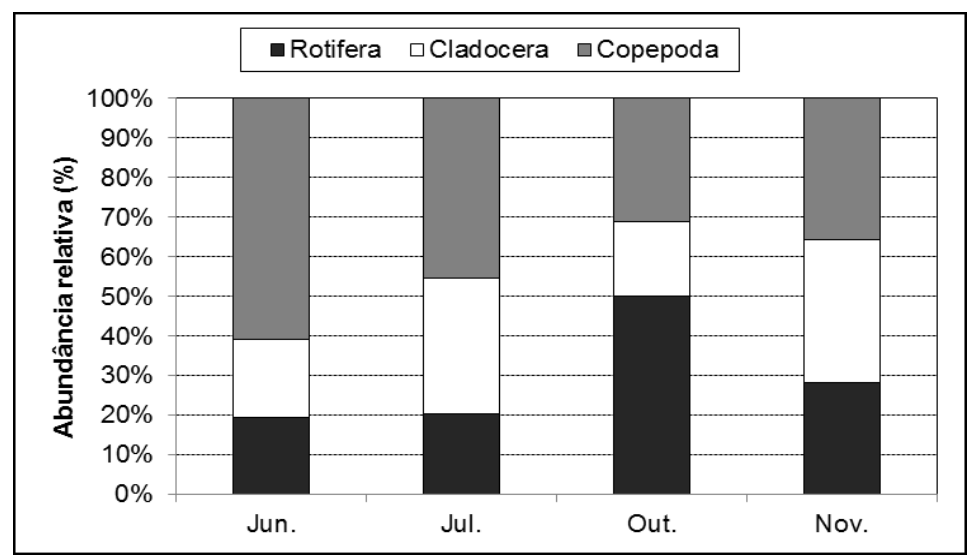

Figura 2- Abundância relativa (\%) da comunidade zooplanctônica do açude Matão no período de junho, julho, outubro e novembro de 2012. 
Entre os Copepoda, houve predomínio de formas jovens (náuplio e copepodito) de Cyclopoida, o que pode estar associado à contínua reprodução desses organismos em regiões tropicais (EDMONDSON, 1959). Durante todo o período de estudo houve maior abundância de Cyclopoida entre os Copepoda. Em julho houve um aumento na densidade de Calanoida, mas a relação Calanoida/Cyclopoida foi inferior a um. Alguns autores (TUNDISI et al., 1988; SENDACZ et al., 1985) tem utilizado este índice Calanoida/Cyclopoida para classificação trófica em ambientes aquáticos. Segundo Reid, (1985) as espécies de Calanoida são planctônicas, assim, as baixas proporções de Calanoida no estudo, provavelmente correspondem à baixa profundidade do corpo d'água que dificulta o desenvolvimento desses organismos.

\section{CONCLUSÃO}

Os resultados obtidos comprovam que o pequeno corpo d'água abriga uma elevada diversidade de espécies mesmo sofrendo pressões antrópicas, como a utilização de água para suprir as necessidades da agropecuária local. A colonização por macrófitas aquáticas no ambiente estudado proporcionou uma maior heterogeneidade ambiental contribuindo significativamente para a diversidade de espécies. Embora esse ecossistema tenha ocorrência de espécies típicas de ambientes com maior grau de trofia, são de grande importância os esforços na conservação deste ambiente, que abriga uma alta riqueza de espécies.

\section{REFERÊNCIAS}

BARBOSA, P. M. M. et al. Zooplâncton (Copepoda, Rotifera, Cladocera and Protozoa: Amoeba Testacea) de lagos naturais do trecho médio da bacia do Rio Doce, Minas Gerais, Brasil. Biota Neotropica, v. 14, n. 1, p. 1-20, 2014.

BEAVER, J. R. et al. The late summer crustacean zooplankton in western U.S.A reservoirs reflects ecoregion, temperature and latitude. Freshwater Biology, v. 59, p. 1173-1186, 2014.

BONECKER, C. C.; LANSAC-TÔHA, F. A.; STAUB, A. Qualitative study of rotifers in different environments of the High Paraná River flooplain (MS), Brazil. Revista Unimar, v.16, p.1-16, 1994.

CABRAL, J. B. P. et al. Concentração de Sedimentos em Suspensão: Reservatório de Cachoeira Dourada - GO/MG, Brasil. Mercator, v. 8, n. 16, p. 233-253, 2009. 
CASTILHO-NOLL, M. S. M. et al. Fauna de invertebrados planctônicos e fitófilos em ambientes lênticos associados a fragmentos florestais remanescentes da região noroeste do estado de São Paulo. In: O. Necchi Junior.(ed.). Fauna e Flora de Fragmentos Florestais Remanescentes da Região Noroeste do Estado de São Paulo. São José do Rio Preto: Departamento de Botânica da Unesp., 2012. v. 1, p. 138-154.

CASTILHO-NOLL, M. S. M. et al. Copépodes (Crustacea, Maxillopoda) em reservatórios rasos . Acta Limnologica Brasiliensia v. 24, n. 2, p.149-159, 2012.

COSTA, M. R. C. et al. Biodiversidade em Minas Gerais: um atlas para a sua conservação. Belo Horizonte: Fundação Biodiversitas, 1998.

DAVIES, B. R. et al. A comparison of the catchment sizes of rivers, streams, ponds, ditches and lakes: implications for protecting aquatic biodiversity in an agricultural landscape. Hydrobiologia, v. 597, p. 7-17, 2008.

DE MEESTER et al. Ponds and pools as model systems in conservation biology, ecology and evolutionary biology. Aquatic Conservation: Marine and Freshwater Ecosystems, v. 15, p. 715725, 2005.

DE MELO, R.; HEBERT, P. D. N. A taxonomic reevaluation of North American Bosminidae. Can. J. Zool., v. 72, p. 1808-1825, 1994.

DECLERCK, S. et al. Ecological characteristics of small farmland ponds: associations with land use practices at multiple spatial scales. Biological Conservation, v. 131, n. 4, p. 523-532, 2006.

DICKERSON, K. D.; MEDLEY, K. A.; HAVEL, J. E. Spatial variation in zooplankton community structure is related to hydrologic flow units in the Missouri River, USA. River Research and Applications, v. 26, p. 605-618, 2010.

EDMONDSON, W. T. Freshwater biology. 2nd ed. New York: JohnWiley \& Sons,1959.

ELMOOR-LOUREIRO, L. M. A. Cladóceros fitófilos (Crustacea, Anomopoda and Ctenopoda) do vale do Rio Paranã, Goiás, Brasil. Revista Brasileira de Zoologia, v. 24, n. 2, p. 344-352. 2007.

. Manual de identificação de cladóceros límnicos do Brasil. Brasília: Universa, 1997.

Cladóceros do Brasil: Famílias Chydoridae e Eurycercidae. Disponível em: $<$ http://cladocera.wordpress.com/>. Data de acesso: 25 de jun. 2014.

GOLTERMAN, H. L.; CLYMO, R. S.; OHNSTAD, M. A. M. Methods for physical and chemical analysis of freshwaters. $2^{\mathrm{a}}$. ed. Oxford: Blackwell Scientific Publications, 1978.

HAVENS, K. E. Summer zooplankton dynamics in the limnetic and littoral zones of a humic acid lake. Hydrobiologia, v. 215, n. 1, p. 21-29, 1991.

HENRY, R. et al. Interações Hidrológicas entre Lagoas Marginais e o rio Paranapanema na sua zona de desembocadura na Represa de Jurumirim. In: (Org.). M.G. Nogueira, A. Jorcin and R. Henry, Ecologia de Reservatórios: Impactos potenciais, Ações de Manejo e Sistemas em Cascata. São Carlos: RiMa. p. 57- 82, 2005.

KOSTE, W. Rotatória- Die Rädertieri Mitteleuropas. I Texband. Berlim: Gebrüder Borntreger. 1978.

KOTOV, A. A.; DUMONT, H. J. Analysis of the llyocryptus spinifer-species group (Anomopoda, Branchiopoda), with description of a new species. Hydrobiologia, v. 428, p. 85-113, 2000. 
LEÓN, D. et al. Zooplankton richness in farm ponds of Andalusia (southern Spain). A comparison with natural wetlands. Limnetica, v. 29, n. 1, p. 153-162, 2010.

LIMA, A. F. et al. Composition and abundance of Cladocera (Crustacea) assemblages associated with Eichhornia azurea (Swartz) Kunyh stands in the Upper Paraná River floodplain. Maringá, v. 25, n. 1, p. 41-48, 2003.

MAIA-BARBOSA, P.M.; PEIXOTO, R. S.; GUIMARÃES, A. S. Zooplankton in littoral waters of a tropical lake: a revisited biodiversity. Brazilian Journal of Biology, v. 68, n. 4, Suppl., p. 1069-1078, 2008.

MAJER, J. D. Dealing with data from extensive invertebrates surveys. In: J.D Majer (ed.). The role of invertebrates in conservation and biological survey. Western Australia: Department of Conservation and Land Management, 1987.p. 53-64.

MARGALEF, R. Limnología. Edição Omega, Barcelona, 1983.

MASSICOTTE, P. et al. Riverscape heterogeneity explains spatial variation in zooplankton functional evenness and biomass in a large river ecosystem. Landscape Ecol, v. 29, p. 67-79, 2014.

MATSUMURA-TUNDISI, T. et al. Eutrofização da represa de Barra bonita: estrutura e organização da comunidade de Rotifera. Brazilian Journal of Biology, v. 50, n. 4, p. 923-935, 1990.

MELLO, C. R. et al. Modelos matemáticos para predição da chuva de projeto para regiões do Estado de Minas Gerais. Revista Brasileira de Engenharia Agrícola e Ambiental, v. 7, n. 1, p. 121-128, 2003.

MERRIAM, G. Corridors and connectivity: animal populations in heterogeneous environments, In: SAUNDERSAND, D.; HOBBS, R. (ed.). Nature Conservation 2: The Role of Corridors. Australia: Surrey Beatty \& Sons, 1991. p.133-142.

NEVES, G. P. et al. Relations among planktonic rotifers, cyclopoid copepods, and water quality in two Brazilian reservoirs. Latin American Journal of Aquatic Research, v. 41, n. 1, p. 138-149, 2013.

NEVES, I. F. et al. Zooplankton community structure of two marginal lakes of the river Cuiabá (Mato Grosso, Brazil) with analysis of rotifer and cladocera diversity. Brazilian Journal of Biology, v. 63, n. 2, p. 329-343, 2003.

NUNES, M. A. et al. Composição e abundância do zooplâncton de duas lagoas do Horto Florestal Dr. Luiz Teixeira Mendes, Maringá, Paraná. Acta Limnológica Brasiliensia, n. 8, p. 207-220, 1996.

PALIK, P. B. et al. Seasonal pond characteristics across a chronosequence of adjacent forest ages in northern Minnesota, USA. Wetlands, v. 21, p. 532-542, 2001.

REID, J. W. Chave de identificação e lista de referências bibliográficas para as espécies continentais sulamericanas de vida livre da ordem Cyclopoida (Crustacea, Copepoda). Bolm. Zool., v. 9, p. 17143,1985

ROCHA, O. et al. Espécies invasoras em águas doces: estudo de caso e propostas de manejo em São Carlos. São Carlos: EdUFSCar, 2005.

SANTOS, M. G.; CUNHA-SANTINO, M. B.; BIANCHINI-JÚNIOR, I. Alterações espaciais e temporais de variáveis limnológicas do reservatório do Monjolinho (Campus da Ufscar). Oecologia Aust., v. 13, n. 3, p. 682-696, 2011. 
SANTOS-WISNIEWSKI, M. J. et al. O estado atual do conhecimento da diversidade dos Cladocera (Crustacea, Branchiopoda) nas águas doces do estado de Minas Gerais. Biota Neotropica, v. 11, n. 3, p. 287-301, 2011.

SANTOS-WISNIEWSKI, M. J. et al. Cladocera Chydoridae of High Altitude Water Bodies (Serra da Mantiqueira), in Brazil. Brazilian Journal of Biology, v. 62, n. 4A, p. 681-687, 2002.

SANTOS-WISNIEWSKI, M. J. et al. Species richness and geographic distribution of the genera Chydorus and Pseudochydorus (Cladocera, Chydoridae) in São Paulo State. Biota Neotropica, v. 8, n.1, p. 21-23, 2008.

SENDACZ, S.; KUBO, E.; CESTAROLLI, M. A. Limnologia de reservatórios do sudeste do Estado de São Paulo, Brasil. VIII. zooplâncton. Bolm. Inst. Pesca, v. 12, n. 1, p. 187-207, 1985.

SEGERS, H. Annotated checklist of the rotifers (Phylum Rotifera), with notes on nomenclature, taxonomy and distribution. Zootaxa, v. 1564, p. 1- 104, 2007.

The biogeography of littoral Lecane Rotifera. Hydrobiologia, v. 323, n. 3, p. 169-197, 1996.

The Lecanidae (Monogononta). In: H.J.F. Dummont (Coord.). (ed.). Guides to the Identification oh the Microinvertebrates of the Continental Waters of the World. vol 2. Netherlands: SPB Academic Publishing, 1995. p. 102.

SERAFIM, M. JR. et al. Rotifers of the Upper Paraná River floodplain:additions to the checklist. Brazilian Journal of Biology, v. 63, n. 2, p. 207-212, 2003.

SINEV, A. Y.; ELMOOR-LOUREIRO, L. M. A. Three new species of chydorid cladocerans of subfamily Aloninae (Branchiopoda: Anomopoda: Chydoridae) from Brazil. Zootaxa, v. 2390, p. 1-25, 2010.

SMINORV, N. N. Fauna of the USSSR. Crustacea: Chydoriadae. Israel Program for Scientific Translation., v. 1, n. 2, p. 1-644, 1974.

SØNDERGAARD, M.; JEPPESEN, E.; JENSEN, J. P. Pond or lake: does it make any difference? Acta Hydrobiologica, v.162, n. 2, p.143-165, 2005.

SOUSA, F. D. R.; ELMOOR-LOUREIRO, L. M. A. Cladóceros fitófilos (Crustacea, Branchiopoda) do Parque Nacional das Emas, estado de Goiás. Biota Neotropica, v. 8, n. 1, p. 159-166, 2008.

SOUSA, F. D. R.; ELMOOR-LOUREIRO L. M. A.; MENDONÇA-GALVÃO, L. Cladóceros (Crustacea, Anomopoda e Ctenopoda) do Cerrado do Brasil Central: Inventário da comunidade associada à macrófitas em zonas úmidas naturais. Biota Neotropica, v.13, n. 3, p. 222-229, 2013.

SYMONS, C. C. et al. Spatial, Environmental, and Biotic Determinants of Zooplankton Community Composition in Subarctic Lakes and Ponds in Wapusk National Park, Canada. Arctic, Antarctic, and Alpine Research, v. 46, n. 1, p. 159-190, 2014.

TEIXEIRA, C.; TUNDISI, J. G.; KUTNER, M. B. Plankton studies in a mangrove IV: size fraction of phytoplankton. Blom. Inst. Oceanogr. v. 26, p. 39-42, 1965.

TOWNSEND, C. R.; BEGON, M.; HARPER, J. L. Fundamentos em Ecologia. 3 ed. Porto Alegre: Artmed, 2010.

TUNDISI, J.G. Água no século XXI: enfrentando a escassez. São Carlos: RiMa, 2003.

TUNDISI, J. G. et al. Comparação do estado trófico de 23 reservatórios do Estado de São Paulo: Eutrofização e manejo. In: TUNDISI, J.G. (ed.) Limnologia e manejo de represas. São Paulo: Série Monografias em Limnologia/USP, 1988. p. 165-204. 
VAN DONK, E.; VAN DE BUND, W. J. Impact of submerged macrophytes including charophytes on phyto- and zooplankton communities: allelopathy versus other mechanisms. Aquatic Botany, v. 72, p. 261-274, 2002.

VAN ONSEM, S.; DE BACKER, S.; TRIEST, L. Microhabitat-zooplankton relationship in extensive macrophyte vegetations of eutrophic clear-water ponds. Hydrobiologia, v. 656, p. 67-81, 2010.

WETZEL, R. G. Limnologia. Lisboa: Fundação Calouste Gulbekian, 1993.

WILLIAMS, P. et al. Comparative biodiversity of rivers, streams, ditches and ponds in an agricultural landscape in Southern England. Biological Conservation, v.115, p. 329-341, 2003.

ZANNATUL, F.; MUKTADIR, A. K. M. A review: potenciality of zooplankton as bioindicator. American Journal of Applied Sciences, v. 6, n. 10, p.1815-1819, 2009. 